\title{
Directly Probing Local Coordination, Charge State and Stability of Single Atom Catalysts
}

Xiaoqing $\operatorname{Pan}^{1}$, Xingxu Yan $^{1}$, Sheng Dai ${ }^{1}$, Mingjie Xu ${ }^{1}$ and George Graham ${ }^{2}$

${ }^{1}$ University of California-Irvine, Irvine, California, United States, ${ }^{2}$ University of Michigan, Ann Arbor, Michigan, United States

Single atom (SA) catalysis is arguably becoming the most active new frontier in catalysis science. Aided by recent advances in practical synthetic methodologies, characterization techniques and computational modeling, a large number of single atom catalysts (SACs) have been found to exhibit exceptional performance for a wide variety of chemical reactions. Visualizing dynamic structure evolution of SACs anchored by various supports in real space offers direct experimental evidence for the understanding of their structure-property relationships. Site-specific information at atomic spatial resolution, obtained by in situ (S)TEM is an essential addition to other in situ techniques (e.g., X-ray photoelectron spectroscopy, $\mathrm{X}$-ray absorption fine structure spectroscopy, et al.), which offer averaged information over larger length scales. In this talk, we present our TEM studies of various catalyst systems to highlight the advantages of TEM on catalysis research: (1) the dynamic evolution of pre-treatment of $\mathrm{Pt} / \mathrm{TiO}_{2} \mathrm{SAC}$ captured by in situ atmospheric aberration-corrected scanning transmission electron microscopy (AC-STEM); (2) determination of charge state of Pt SAs by advanced electron energy-loss spectroscopy (EELS); (3) experimental and theoretical understanding of the local coordination of single atom catalysts on twodimensional (2D) material supports.

We employed in situ AC-STEM at atmospheric pressure to examine the mobility of Pt atoms on the $\mathrm{TiO}_{2}$ support. As shown in Fig.1, the Pt atom remained at the same location on the $\mathrm{TiO}_{2}$ support during the exposure to mild oxidation $\left(300{ }^{\circ} \mathrm{C}, 760\right.$ Torr of $\left.\mathrm{O}_{2}\right)$ and mild reduction $\left(250{ }^{\circ} \mathrm{C}, 760\right.$ Torr of $\left.5 \% \mathrm{H}_{2}\right)$ conditions, respectively. However, following $30 \mathrm{~min}$ of harsh reduction (exposure to760 Torr of $5 \% \mathrm{H}_{2}$ at $450{ }^{\circ} \mathrm{C}$ ), the $\mathrm{Pt}$ atom moved about $1.6 \mathrm{~nm}$, reflecting the evolution of its anchoring site and local coordination, and greatly improves the activity of $\mathrm{CO}$ oxidation of the $\mathrm{Pt} / \mathrm{TiO}_{2} \mathrm{SAC}$, which is further demonstrated by the ex situ activity test [1].

In addition, the stability of the supported single metal atom is strongly dependent on its charge states, which is also crucial to elucidate the reaction mechanism. EEL spectra of $\mathrm{Pt} N$ edges were used to interrogate the charge state of the $\mathrm{Pt}_{\text {iso }}$ species on various oxide supports. On the basis of a comparison to reference spectra, the charge state of Pt SA can be experimentally distinguished. For $\mathrm{Pt}_{\mathrm{iso}} / \mathrm{CeO}_{2}$ catalysts, it was concluded that the oxidation state of this $\mathrm{Pt}_{\text {iso }}$ species absorbed on (111) surface facets of $\mathrm{CeO}_{2}$ is +4. This agrees with the expected charge state of the Ptiso species following oxidation that has been predicted and observed in model system studies, and the XAS measurements [2].

Furthermore, integrating SAs with structurally well-defined 2D 1T-MoS2 support enables quantitative identification of SA's anchoring sites, coordination environments, and bonding information [3]. Combining low-voltage AC-STEM imaging and first-principles calculations, the local coordination and anchoring structure of single atom are unambiguously identified in several catalysts. Fig. 2(a) displays the atomic resolution STEM image of $\mathrm{Zn} / 1 \mathrm{~T}-\mathrm{MoS}_{2}$ sample at $60 \mathrm{kV}$. Atomic column \#3 of the experimental 
line profile of ADF intensity in Fig. 2(b) is obviously brighter than surrounding atom columns, indicating the existence of the SA in that position. Compared to simulated images of two different structures in Figs. 2(c) and 2(d), the observed SA was assigned to the absorbed Zn model, which was predicted by firstprinciples calculations. The experimental intensity ratio between \#3 and an average of surrounding Mo positions is 1.95 , which is consistent with the simulation value (2.05) of the absorbed model [4].
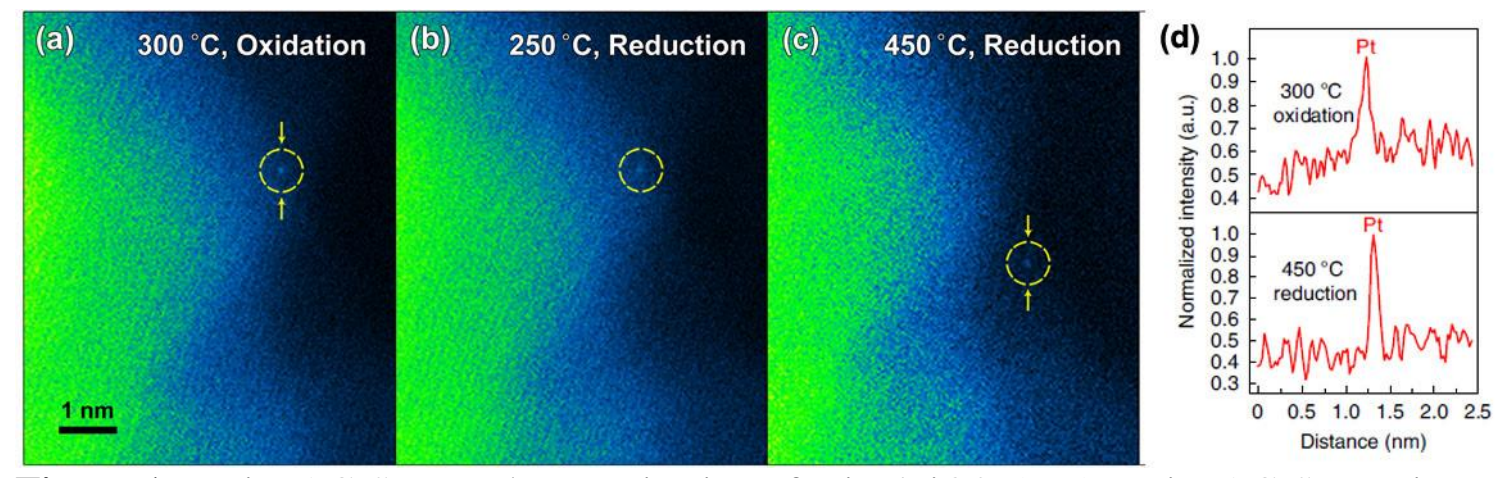

Figure 1. In situ AC-STEM characterization of Ptiso/TiO2. (a-c) In situ AC-STEM images of Ptiso/TiO2 after $30 \mathrm{~min}$ at different annealing conditions: $300{ }^{\circ} \mathrm{C}, 760$ torr of $\mathrm{O} 2$ for $30 \mathrm{~min}$ (a); $250{ }^{\circ} \mathrm{C}, 760$ torr of $5 \% \mathrm{H} 2$ (balanced with Ar) for $30 \mathrm{~min}(\mathrm{~b}) ; 450{ }^{\circ} \mathrm{C}, 760$ torr of $5 \% \mathrm{H} 2$ (balanced with Ar) for 30 min (c). The yellow circles identify the same Pt single atom. A false-coloring scale was used in (a-c) to enhance contrast on the Pt atom. (d) Intensity profile of a line scan (along the yellow arrows) shown in (a) and (c) normalized to the identified Pt atom.
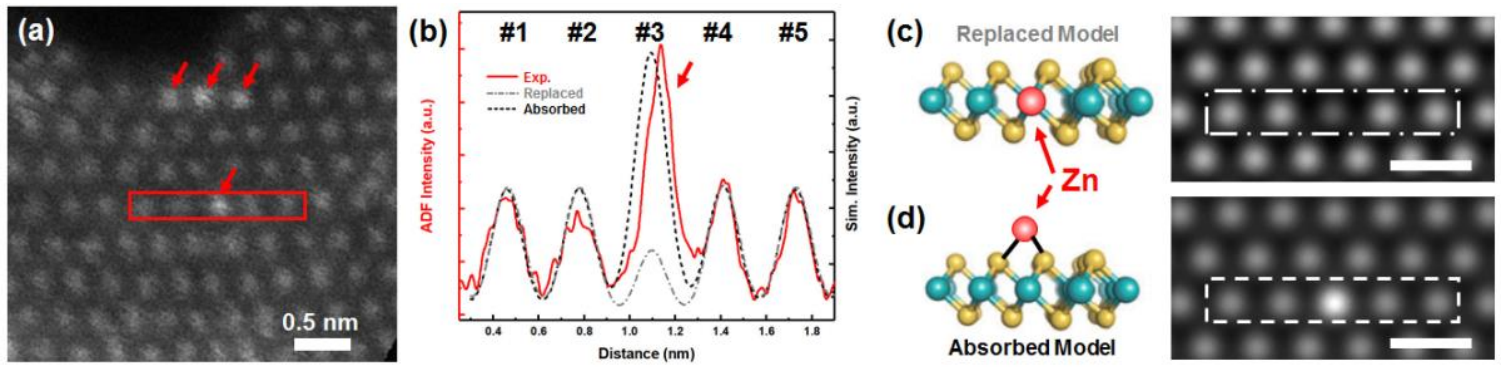

Figure 2. Quantitative identification of bonding structure of Zn SA on 1T-MoS2 support. (a) Atomic resolution annular dark-field (ADF) STEM image of Zn/1T-MoS2 at $60 \mathrm{kV}$. The red arrows point to the locations of Zn SAs. (b) Comparison of intensity profiles from the experimental image and two simulated images of replaced and absorbed models respectively. (c) Side view of the atomic structure of replaced $\mathrm{Zn}$ model and associated simulated STEM image. The yellow, green and red balls are S, Mo and Zn atoms respectively. (d) Side view of the atomic structure of absorbed $\mathrm{Zn}$ model and associated simulated STEM image. Scale bars in (a, c, d) are all $0.5 \mathrm{~nm}$.

\section{References}

[1] L. DeRita, et al. Nat. Mater. 746 (2019), p.746

[2] J. Resasco et al., J. Am. Chem. Soc. 142 (2020), p. 169-184.

[3] Y. C. Huang et al., Nat. Commun. 10 (2019), 982. [4] The authors acknowledge the support of the University of California Irvine Materials Research Institute for the use of TEM facilities. 